\title{
Criminal Community Detection Based on Isomorphic Subgraph Analytics
}

https://doi.org/10.1515/comp-2020-0112

Received Dec 15, 2019; accepted Mar 29, 2020

\begin{abstract}
All highly centralised enterprises run by criminals do share similar traits, which, if recognised, can help in the criminal investigative process. While conducting a complex confederacy investigation, law enforcement agents should not only identify the key participants but also be able to grasp the nature of the inter-connections between the criminals to understand and determine the modus operandi of an illicit operation. We studied community detection in criminal networks using the graph theory and formally introduced an algorithm that opens a new perspective of community detection compared to the traditional methods used to model the relations between objects. Community structure, generally described as densely connected nodes and similar patterns of links is an important property of complex networks. Our method differs from the traditional method by allowing law enforcement agencies to be able to compare the detected communities and thereby be able to assume a different viewpoint of the criminal network, as presented in the paper we have compared our algorithm to the well-known GirvanNewman. We consider this method as an alternative or an addition to the traditional community detection methods mentioned earlier, as the proposed algorithm allows, and will assists in, the detection of different patterns and structures of the same community for enforcement agencies and researches. This methodology on community detection has not been extensively researched. Hence, we have identified it as a research gap in this domain and decided to develop a new method of criminal community detection.
\end{abstract}

Keywords: Criminal Network Analysis; Algorithm for graph isomorphism, Social Network, Cyber Security, Graph analytics

\footnotetext{
*Corresponding Author: Theyvaa Sangkaran: School of Computer Science and Engineering (SCE), Taylor's University, Subang Jaya, Selangor, Malaysia; Email: theyvaasangkarankrishnan@sd.taylros.edu.my
}

\section{Introduction}

The modernised science of networks has substantially advanced our perception of complicated of systems. An example for one of the applicable characteristics of graphs is the ability to perform representation of real systems such as community structure, or clustering, i.e. the organisation of nodes in a community. Such clusters, or communities, can be seen as a stand-alone bay of graph theory, similarly, for example, the internal representation of the human tissues or the organs in the human body. Detecting communities is of great importance in sociology, biology, and computer science domains where systems are oftentimes represented as graphs [1].

Various researches have validated that these communities are made up of a group of entities, or people in the case of human networks, which exist in various concepts or forms. These types of forms or concepts may be an organised criminal network, collaboration of (academic) researchers, group of students, chemical bonds etc. Hence, detecting communities becomes non-trivial amidst the fact that theoretical research is being carried out on how to devise effective and efficient algorithms for this purpose. Likewise, practical implementations of the developed algorithms are being executed for the associated merit of its utilization in society. Community detection simply means finding the clusters for proper identification of a group of people. Communities in networks do overlap most of the time as nodes that can belong to multiple communities at a time. In order to identify such overlapping communities [2], it is very important to understand the structure of a community as well as the function of a realworld network.

Through extensive literature review, it can be determined that due to an inherent feature of all known com-

*Corresponding Author: NZ Jhanjhi: School of Computer Science and Engineering (SCE), Taylor's University, Subang Jaya, Selangor, Malaysia; Email: noorzaman.jhanjhi@taylors.edu.my

Azween Abdullah: School of Computer Science and Engineering (SCE), Taylor's University, Subang Jaya, Selangor, Malaysia; Email: azween.abdullah@taylors.edu.my

ə Open Access. ๑ 2020 T. Sangkaran et al., published by De Gruyter. (cc) BY 4.0 License This work is licensed under the Creative Commons Attribution 
munity detection algorithms, i.e. their hierarchical nature, the method often used in detecting a community or communities can either be agglomerative or divisive, as shown by [8]. Concisely put, the agglomerative method of clustering, also known as the 'bottom up' approach, works to initiate the partitioning of a network from a singleton (i.e. one single node) moving up the structural hierarchy, which is present in the network and which then combines with other nodes causing, at each step, the merging of increasingly larger areas of the graph until the whole graph or its components are being merged together.

On the other hand, the divisive clustering process, which is a 'top down' approach, works to initiate the partitioning of a network by considering the complete network as a single module and then recursively splitting the root nodes as it moves downwards through the hierarchical structure present in the given network., i.e. it disassembles a complete network down to a single node. In a broader scope, all community detection algorithms, as reviewed by [3], fall under the following, namely: direct partitioning, skeleton clustering method, leadership expansion, clique percolation, division algorithm, agglomeration hierarchical clustering, matrix blocking and label propagation. Moreover, all these algorithms can either be used for detecting overlapping communities or disjoint communities.

Figure 1 shows the communities detected from a simple graph. Note that some nodes have inter-connectivity with other communities. With respect to the relationships among them, it represents strongly linked sub-networks from the entire network, which can also represent the key participants and key subgroups. This can be purposeful, meaningful, usable, valuable and actionable information from complex networks. The community structure holds important information that is being mined through com-

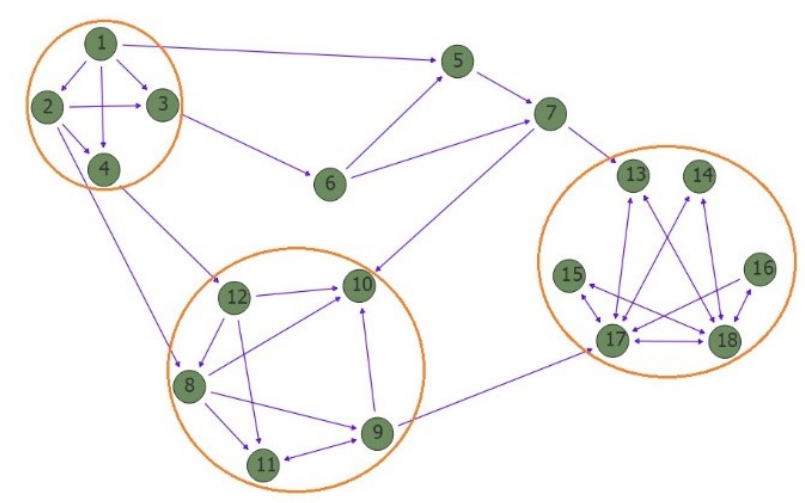

Figure 1: A simple graph with three communities detected, enclosed by the circles. munity detection mechanisms and is now a dominant research area since many real-world problems can be depicted, formulated and solved with the use of graph and its analyses. Through community detection, the internal network organisation of a given network is uncovered and proper understanding of the characteristics inherent to the dynamic processes specific to the network is fostered.

The major contribution of this research study is the design, implementation, evaluation, and validation of a novel algorithm for detecting communities in a graph network. This research undertaking's novelty lies in the development of an algorithm that detects communities and finds isomorphic communities within a given graphical network.

Section 2 of this paper concisely tackles the fundamental theories of the novel algorithm and presents a review of the related literature. Section 3 discusses the algorithm, necessary mathematical notations, and applicable procedures. Section 4 of this paper talks about the implementation and evaluation of the novel algorithm and of some baseline community detection algorithms for the purpose of comparative analysis. Lastly, Section 5 presents the results and discussion, conclusion and future lines of work.

\section{Related Works}

This section contains the comprehensive review of criminal network analysis, community detection graph and its types, graph theory and its components and isomorphic graph algorithms and community detection algorithms.

\subsection{Isomorphic Graph Algorithm}

Our previous research undertaking [4] presented an extensive survey of isomorphic graph algorithms usable for graph analytics. Basically, isomorphic (sub)graph algorithms are used for matching two graphs by ascertaining the mapping associated with nodes of the involved graphs. The existence of different types of mapping is the result of how different types of constraints can be applied on the mapping, further leading to graph-subgraph isomorphism, monomorphism, automorphism, or strict isomorphism.

Over the years, various algorithms for determining isomorphism between graphs have been designed and implemented as well as comparatively evaluated. Some of these algorithms include the Ullman Algorithm [5], VF [6] and VF 2 [7] algorithm, Schmidt and Druffel method [8], Corneil 


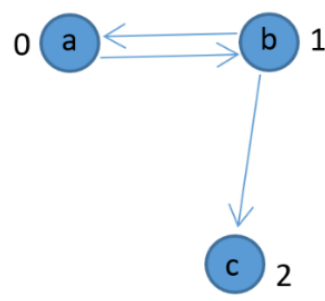

Figure 2: An example of a query graph.

and Gotlieb [9] method, and Merging Lexicographic Chain (MLC) [10] algorithm. These algorithms have several varying underlying mathematical theories and concepts for determining isomorphism between graphs. Moreover, as we uncovered in our previously published study, each of the highlighted isomorphic graph algorithms possesses its strengths and weaknesses [4].

Table 1 shows a simplified results of query graph matching. presents an example of a query graph, while Figure 3 depicts its matches via simple, dual and isomorphism in a data graph. Simple matches are denoted by an ' $s$ ', and isomorphic matches by an ' $i$ '. Table 1 summarized the matches.

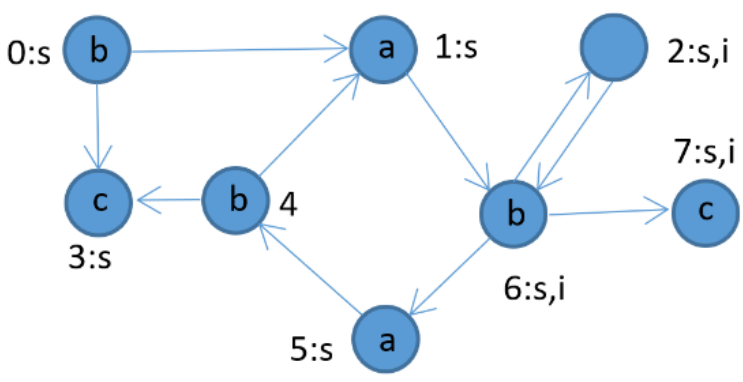

Figure 3: An example of matched nodes of the query graph.

Table 1: A simplification of the results of query graph matching.

\begin{tabular}{cc}
\hline $\begin{array}{c}\text { Query Nodes } \\
\text { (The node we are trying to } \\
\text { find the isomorphic match) }\end{array}$ & $\begin{array}{c}\text { Matched Nodes } \\
\text { (The node which meets the } \\
\text { isomorphic criteria of } \\
\text { queried node) }\end{array}$ \\
\hline 0 & $2: \mathrm{s}, \mathrm{i}$ \\
1 & $6: \mathrm{s}, \mathrm{i}$ \\
2 & $7: \mathrm{s}, \mathrm{i}$ \\
\hline
\end{tabular}

The other nodes $(3,4,5)$ have no isomorphic match.

\subsection{Community Detection Algorithm}

Community detection is an important aspect of Social Network Analysis (SNA) as it decomposes a complex graphical network into smaller units for deeper analysis of relationships between nodes. Though communities are without quantitative definition, is it often referred to as a group of nodes with densely connected intra-group modes. The hidden nature of communities within a given network drives the continuous development of community detection algorithm having optimal speed for community detection completion as well as efficiency in the area of computational cost reduction. In simple words, discovering and splitting up a group of complex nodes of a graphical network into groups of smaller densely connected intra-group nodes is referred to as community detection.

Community detection in a graphical network is carried out using several algorithms including, but not limited to, Random Walk, Clique Percolation Method (CPM), Link Graph and Link Partitioning, Simulated Annealing Fuzzy Detection, Local Expansion and Optimisation, Statistical Inference based methods, Extremal Optimisation, NMF and PCA based methods, Spin Models, Partitional Clustering, Graph Partitioning, Hierarchical Clustering, Spectral Clustering, Greedy Optimisation, Genetic Algorithms and Spectral Optimisation as revealed, by [11-13].

The various community detection algorithms highlighted above are used to either detect overlapping communities or disjoint communities. The community detection algorithms used for the detection of disjoint communities are usually categorised into three vis-a-vis traditional, dynamic, modularity-based algorithms. Each method is based on different mathematical theories, is used in different practical applications and always outperforms other methods in their application. Many of the aforementioned community detection algorithms have not yet been implemented in, and evaluated on, a criminal network, thus making this research study a pivotal work on the topic of community detection in a typical criminal graphical network. Figure 4 below is an illustration of Taxonomy of Community Detection Algorithms.

Popular community detection algorithms used for SNA include Factorised Asymptotic Bayesian (FAB) inference with Belief Propagation, an algorithm which strengths lies in having no parameters, effecting consistent results over sparse and dense graph even as its extended version is known to lack consistency based on the usage of the Belief Propagation [14]. Another popular algorithm used for detecting community via SNA is the modularity optimisation and maximum likelihood equivalence algorithm. This algorithm is known to produce a consistent 


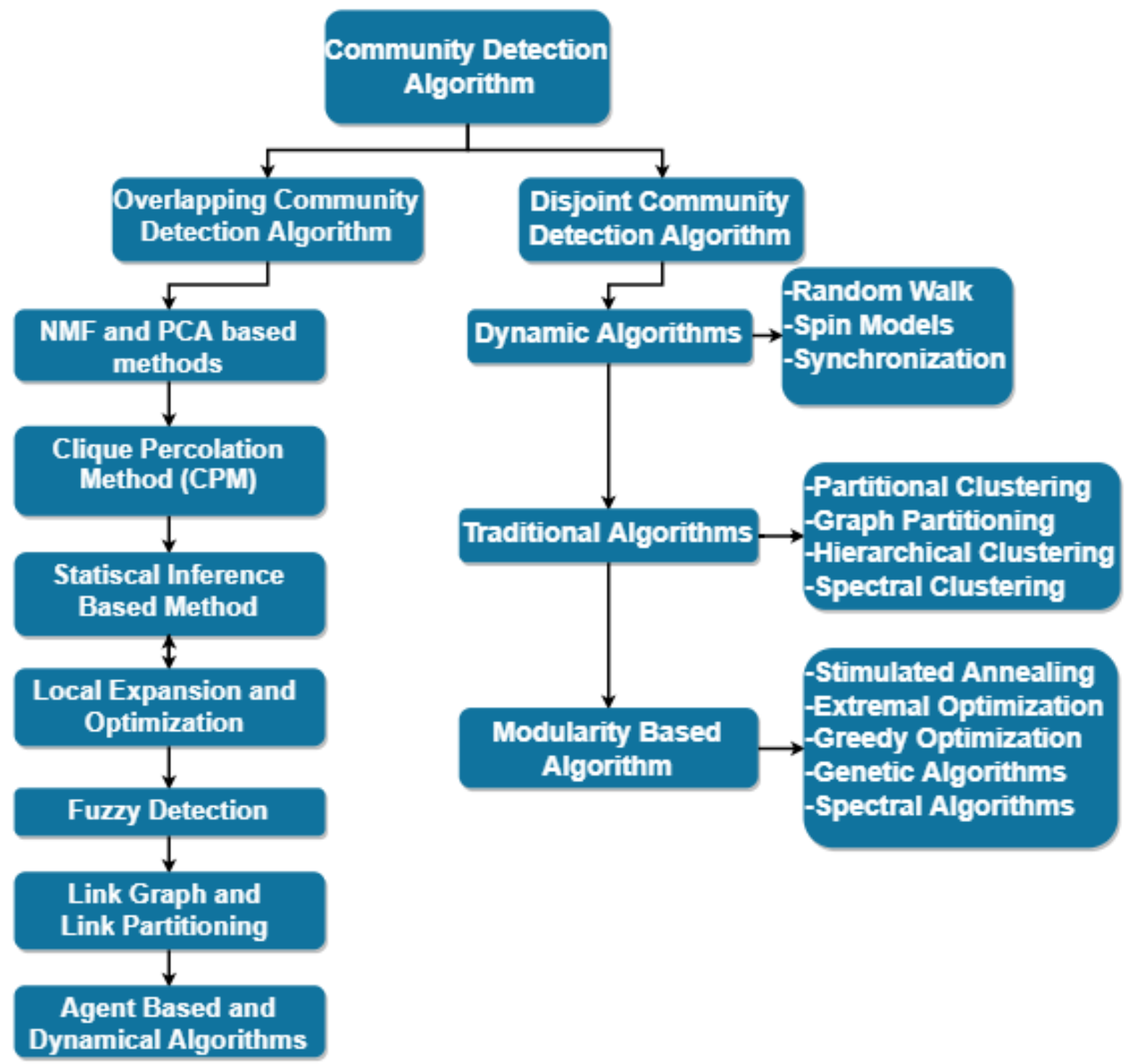

Figure 4: Taxonomy of Community Detection Algorithms.

result in detecting communities when compared against its maximum-likelihood method though it heavily relies on and is only applicable when the resolution parameter value is correct [15].

Yet another popular SNA community detection algorithm is the Minimum Description Length (MDL) principle and multilevel Monte Carlo algorithm. This algorithm can produce a simple, unbiased, efficient and fully nonparametric analysis of large-scale features of a large network and, thus, is able to detect a reasonable number of communities within a given large network [16]. Lastly, Matrix Blocking community detection algorithm is popular in SNA mainly because it does not require specification of clusters nor the clustering of all network nodes. Matrix Blocking is also a popular choice due to its lowered computational cost as well as its applicability on different graph types such as undirected, directed and/or bipartite graphs. Also, the ability of Matrix Blocking method to output singletons as a community serves as the chief limitation of this method. 


\subsubsection{Review of Methodology Used for Community Detection}

Pragmatically, the research work carried out by [16] used a parsimonious method to detect communities within a graph without prior knowledge of the number of communities inherent in the network. Unlike other community detection algorithms that assume the number of clusters in a network beforehand, this research work devised a method for obtaining the unknown number of communities before accordingly proceeding at their detection through the use of the MDL principle for selecting the best optimal model that fits into the given data.

The results obtained through MDL are then supplied to the Monte Carlo module of the algorithm responsible for the safe detection of communities with respect to the garnered results. This hybrid MDL and Monte Carlo algorithm was successfully used in the effective detection of communities from large networks without having to assume the initial number of communities present in the network. The algorithm was implemented and evaluated based on the American football network and the political books graphical network data.

Another similar research work is that of [17] that also does not assume to know the number of communities within a network (though this is known to be quite a difficult problem). In the study, a mathematical principle method was adapted to estimate the ideal number of communities present in a network with the use of the maximum-likelihood mathematical method. Both real-world and computer-generated graphical networks were used to evaluate the community detection algorithm. Maximum-likelihood, as implemented, proved to be a reliable mathematical principle for making a safe inference of the number of communities available for a given graphical network: results showed it is adequately capable of determining the previously unknown number of communities before proceeding to detect the communities. The algorithm was tested on the Karate club network data, college football network data, Les Misérables network data and the Dolphins network data, respectively.

Typical community detection algorithms make use of the hierarchical nature of the network data, and such is the algorithm used in the research work carried out by [18]. A spectral clustering algorithm was implemented for finding communities within a sparse network. Unlike the previously reviewed research studies, this paper presented an algorithm that assumes the number of clusters in a graphical network prior to detecting communities. In order to resolve the limitation of spectral clustering for community detection, the paper presented an enhanced spectral clus- tering algorithm that uses nonbacktracking matrix to solve the woeful performance of traditional clustering that oftentimes fails to detect communities within networks known to have communities.

Using the nonbacktracking matrix, the spectral clustering method permitted the strong separation of bulk eigenvalues from those eigenvalues that really hold vital information about a network's community structure. Evaluation of the enhanced spectral clustering algorithm was carried out and comparatively analysed against Belief Propagation as well as Laplacian, normalised Laplacian, and even random walk matrix methods. Evaluating the algorithm on a (sparse) network of both realword and computer-generated network data-Dolphins, Karate, Polbooks, Football, Adjnoun, Pollblogs network data-demonstrated that the enhanced spectral clustering algorithm is absolutely better than the traditional spectral clustering algorithm and fiercely competed with other highlighted community detection algorithms.

Using modularity Density, [19] presented a study showing that communities were detected for a given graphical network using a different evolution method, Community Detection Based on Differential Evolution by Using Modularity Density (CDDEA). CDDEA was implemented and evaluated using both synthetic and real-world network datasets. When it was compared against traditional modularity-based algorithms, results showed that it was a superior and effective method for partitioning complex networks. The developed algorithm made use of a novel tuning parameter to identify various communities for a given complex network.

\subsubsection{Critical Review of Community Detection in Graphical Criminal Network Orientation}

Meanwhile, few studies on detecting communities in a graphical criminal network have been conducted to date. One of the available research works [? ] detected communities in a typical criminal network by using the shortest paths network search algorithm (SPNSA) and network centrality measure. Having used the Enron email network data, the algorithm present in their work was reported to have outperformed the k-neighborhood detection method. The communities detected using the said algorithm were also reported to be small and sparse, thereby facilitating further probing.

[20] presented Louvain and SpeakEasy methods for detecting communities in a criminal network. The algorithms used were implemented on Cavair and Ndrangheta gang datasets (Stupor Mundi and Chalonero) and it was re- 
ported that stable networks were detected while augmentation of covert links was carried out to facilitate network restoration though further investigation into edge augmentation that was mentioned as a topic of future research $[21,22]$. On the other hand, the study of Calderoni, Piccardi, and Milan in [23] seems to be the oldest research on detecting communities in a criminal network. The research that used maximum-modularity algorithm to detect communities in the Ndrangheta criminal network reported the detection of communities that are very similar to a known criminal gang, with node classification achieved at $90 \%$ precision.

The detection of communities within a criminal network is pivotal to this research undertaking. Thus, a novel method of detecting communities within a criminal network is proposed and discussed in Section 3.

\section{Experimental Setup}

The researcher intends to undertake the research in broad dimensions; first to perform an initial visualization and extraction of the data using some selected open source tools so as to gain a general overview of the criminal network graph, and second to apply the relevant methods and techniques designed such as pruning, and newly developed community detection algorithm with isomorphic feature for addressing and achieving each of the objectives outlined for the research. In this write-up, the terms interactions and links are used interchangeably to mean a relationship. Subgroups and communities are also used in a like manner. The different steps involved in the proposed methodology include the selection of criminal data source. We have chosen to work with the UCINET Cocaine Dealing Natarajan. Data extraction, cleansing, collection and restructuring of some data may be needed due to duplication. Heuristics Community Detection Algorithm based on Isomorphic Subgraph Matching algorithm will be applied to the graph in order to extract isomorphic graph.

The researchers planned to use one of the state-ofart isomorphism algorithms to complete the comparison with their new proposed method. Although some influential isomorphism algorithms, such as VF2 [6], VF3 [22], LAD [23], RI [24] and Nauty algorithm [25], are optimal and can output perfect results in graph isomorphism detection, their computational complexity is not polynomial-time. On the other hand, the Girvan-Newman community detection model technique drastically reduces the running time of the calculation even though the resulting estimate of betweenness necessarily suffers from the statistical fluctua- tions inherent in random sampling methods. Even though Girvan-Newman is computationally intensive, it is very suitable for small graphs, which happen to be the focus of our research, due to the speed with which it can detect a community. As for the datasets used, we utilised a covert network dataset from UCINET, namely Cocaine Dealing Natarajan, which resulted from an investigation into to a large cocaine trafficking organization in New York City. Both experiments were conducted in the same environment. Examples of the metrics used to match the isomorphic graph were degree centrality, closeness centrality and common neighbors.

\section{Proposed 'Criminal Community Detection Algorithm'}

In this section, we explain the principle of the proposed 'Criminal Community Detection Algorithm for Isomorphic Subgraph Matching' (CCDA-ISM) modelled from Ullman Algorithm. Below is the pseudocode of CCDA-ISM algorithm. The refined pseudocode for the algorithm is as follows:

Because $|\mathrm{s}(0)|+\ldots+|\mathrm{s}(|\mathrm{Vq}|-1)| \leq|\mathrm{V}||\mathrm{Vq}|$, the outer while loop in line 3 may execute at most $|\mathrm{V}||| \mathrm{Vq} \mid$ times. This would correspond to a scenario in which only one vertex is removed with each iteration of the loop.

The next two for loops (lines 5-6) execute a total of $|\mathrm{Eq}|$ times, and there are at most $|\mathrm{V}|$ vertices in $|\mathrm{s}(\mathrm{u})|$ for any $\mathrm{u}$, Vq, creating abound on the innermost for loop (line 7). The intersection operation in line 8 takes at most $|\mathrm{V}|$ steps, and so the total time complexity is $\mathrm{O}(|\mathrm{Eq}||\mathrm{Vq}||\mathrm{V}| 3)$. Note that vertex 0 is in $s(1)$ despite the fact that it does not have a vertex in $\mathrm{s}(0)$ as a parent.

Firstly, given a Graph G, and a graph pattern Q, the objective of isomorphic subgraph matching is to identify all sub-graphs mappings, $\mathrm{P}$ from $\mathrm{G}$, that are isomorphic to Q.

Input: pattern $Q$ and graph $\mathrm{G}$

Output: all isomorphic mappings $P$ from $Q$ to $G$

Matching procedure $(\mathrm{P})$

a) if $P$ covers all nodes in $Q$ then output $P$;

b) or else compute the set $\mathrm{S}(\mathrm{P})$ of all candidate pairs for inclusion in $\mathrm{P}$

c) for each pair $\mathrm{p}=(\mathrm{u}, \mathrm{v})$ in $\mathrm{S}(\mathrm{P})$

i) if p passes feasibility check

ii) then $\mathrm{P}^{\prime} \leftarrow \mathrm{P}\{\mathrm{p}\}$; call Match( $\left.\mathrm{P}^{\prime}\right)$;

d) Restore data structures 


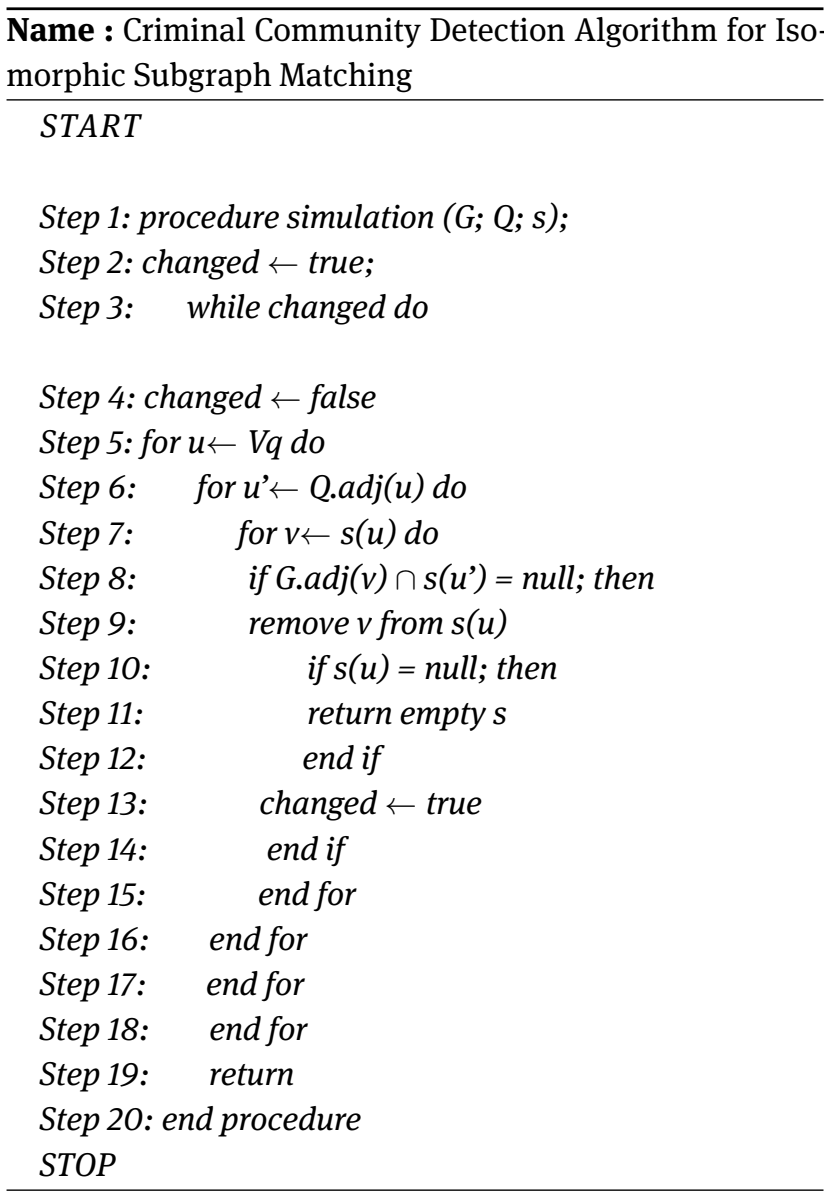

Reiteration refinement

For each pair $\mathrm{p}=(\mathrm{u}, \mathrm{v})$ in $\mathrm{S}(\mathrm{P})$ :

i) enumerate all possible extensions, for refinement

ii) if the feasibility test is not successful, drop it and try the next.

\section{Experimental Results}

As summarised and concluded in Table 2, the experimental results demonstrated that CCDA-ISM can detect communities that overlap with the well-studied Girvan-Newman algorithm. To explain further on this, the proposed CCDAISM managed to identify a matching Community 3 as represented in Figure 7 (nodes 3L,25S,27M) in the community represented by red nodes.

The general term of pruning refers to the pruning cuts made in a certain order, so that the total number of cuts is greatly reduced. The purposes of pruning are varied. Some pruning may be required to train a dataset or to restrict the search area. Other forms of pruning may be required to maintain or improve the state and quality, reliability of

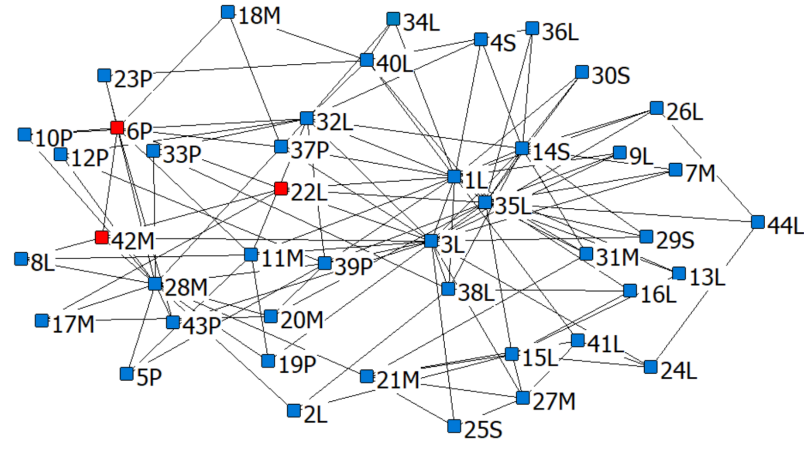

Figure 5: The base subgraph, which is also the input graph for the isomorphic subgraph detection.

the dataset. The pruning procedure used for our subgraph isomorphism algorithm is based on a concept known as graph simulation. The conditions in Ullmann's equation for checking the pruning process of the search space can be adapted to create an algorithm as seen in the pseudocode.

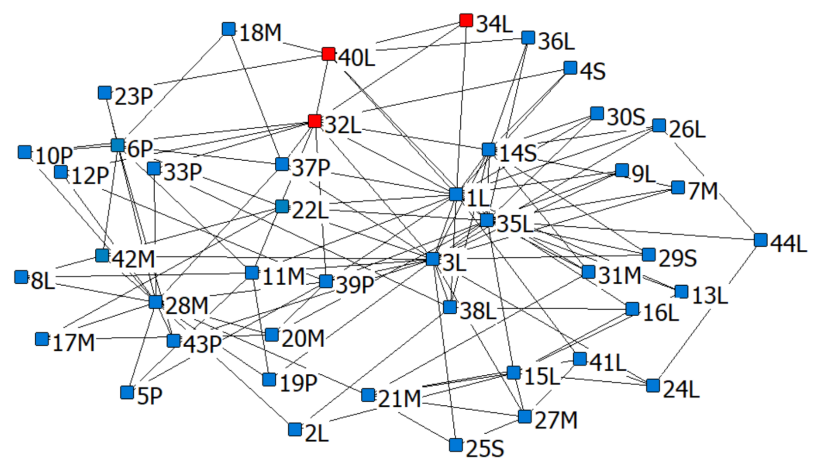

Figure 6: One of the communities (Community 1) detected using the new community detection algorithm.

Complex networks are known to have a structure. In Social Network Analysis and Criminal Network Analysis, the structure are also known as Community Structure. These nodes can be grouped into (potentially overlapping) sets of nodes which are densely connected. Community detection model is based on the notion of edge betweenness centrality that was investigated in the domain of SNA. The betweenness centrality value of an edge is defined as the number of shortest paths between nodes

CCDA-ISM is effective in community detection problems and is superior to traditional community detection techniques because it is able to detect communities that traditional algorithms miss. The fact that some of the detected communities may overlap connotes that the communities detected are correct and the one that does not 
Table 2: A simplified result of the experiment.

\begin{tabular}{|c|c|c|c|c|}
\hline Algorithms & Fig. No & Community Detected & $\begin{array}{l}\text { Isomorphic Community } \\
\text { Detected (P') }\end{array}$ & Remarks \\
\hline Proposed CCDA-ISM algorithm & 5 & Input graph (Q') & Nodes- $(6 P, 28 M, 22 L)$ & Base sub graph (Q') \\
\hline Proposed CCDA-ISM algorithm & 6 & YES - Community 1 & Nodes- $(32 L, 40 L, 34 L)$ & $\begin{array}{l}\text { Matched Isomorphic Subgraph } \\
\text { (Community 1) }\end{array}$ \\
\hline Proposed CCDA-ISM algorithm & 7 & YES - Community 2 & Nodes- $(35 L, 14 S, 31 M)$ & $\begin{array}{l}\text { Matched Isomorphic Subgraph } \\
\text { (Community 2) }\end{array}$ \\
\hline Proposed CCDA-ISM algorithm & 8 & YES - Community 3 & Nodes- $(3 L, 25 S, 27 M)$ & $\begin{array}{l}\text { Matched Isomorphic Subgraph } \\
\text { (Community 3) }\end{array}$ \\
\hline Girvan-Newman algorithm & 9 & YES - 4 key communities & Nodes- $(35 \mathrm{~L}, 14 S, 31 M)$ & $\begin{array}{l}\text { Matched Isomorphic Subgraph } \\
\text { (Overlap with Community 3) }\end{array}$ \\
\hline
\end{tabular}

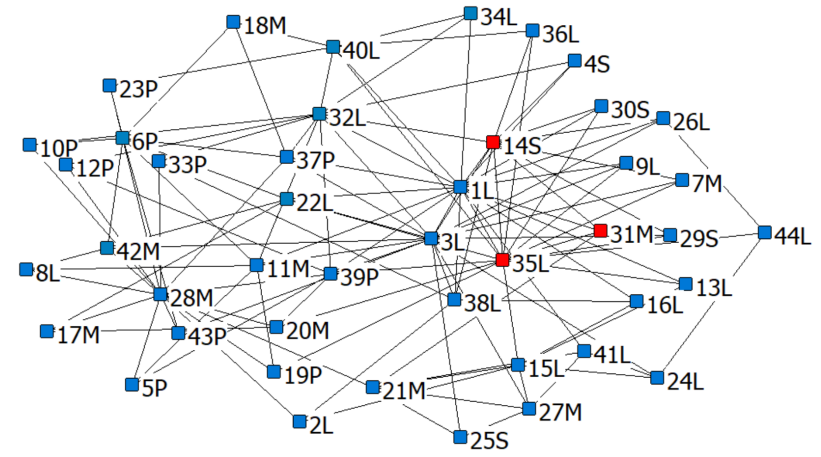

Figure 7: One of the communities (Community 2) detected using the new community detection algorithm.

overlap opens up a new perspective in the domain of community detecting by the incorporation of subgraph isomorphic matching features.

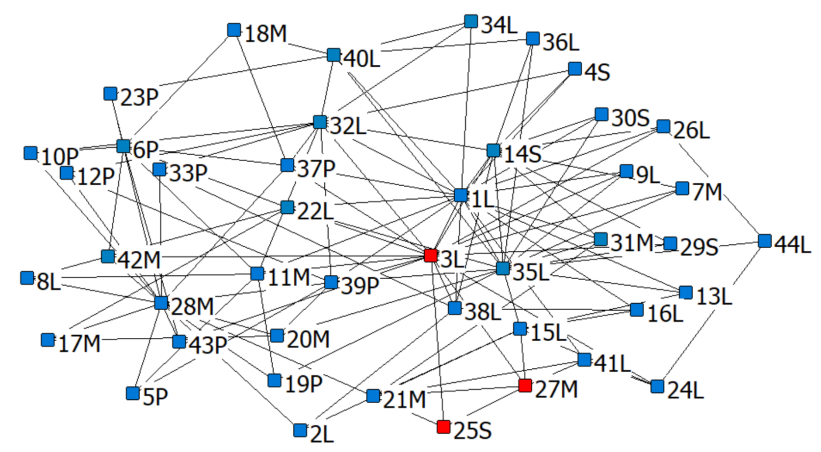

Figure 8: One of the communities (Community 3) detected using the new community detection algorithm

Our algorithm falls into the category of tree-search based algorithms described earlier, with the primary difference lying in the pruning algorithm, an index used for mapping each label to every vertex in $\mathrm{G}$ with that label, thus allowing fast initial access to feasible matches. Our algorithm begins by obtaining feasible matches: for each given query vertex $u$, it creates the set $s(u)$ to contain all vertices in the data graph with the same label as $u$. This is the same as Ullmann's algorithm except that it ignores the degree constraint. Overall, during the experimentation, it turned out that this constraint did not help our algorithm and that in the cases where response times were exceptionally fast, it actually slowed down the algorithm (if only by a few tens of milliseconds). This is attributable to how the pruning process rapidly eliminates many of the vertices that would have been eliminated by an initial degree constraint anyway.

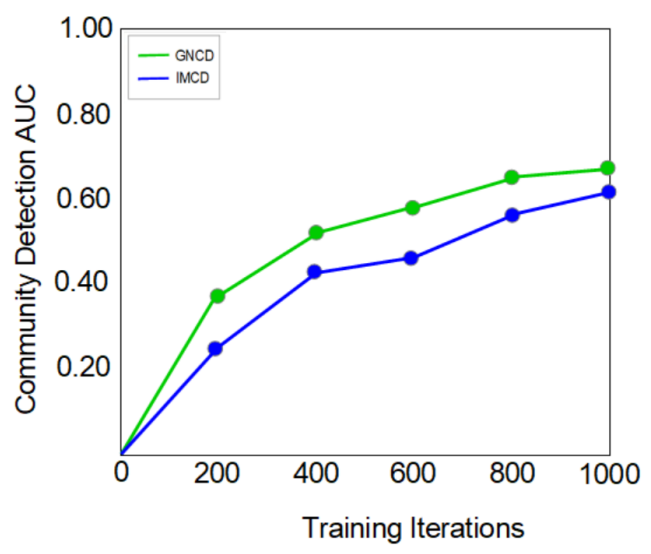

Figure 9: AUC Score

The Area Under Curve (AUC) metric is a type of statistical learning metric that indicates the predictive accuracy of the model and has a value that lies between 0 and 1 , where a higher value indicates a better predictive accuracy. The AUC scores seem to indicate as the number of iterations of training is performed, the IMCD model seems to achieve a level of accuracy nearer to the GNCD model. This could partly be affected by the initial base sub-graph of using just three nodes in the training of the IMCD model 
within the limit of 1000 iterations. As per the results in the table below, the IMCD model seems to use a significant less time for training purposes to achieve about the same AUC scores. The IMCD may provide a complementary perspective of communities detected compared with models developed on classical algorithms such as Girvan-Newman, which uses more computing time.

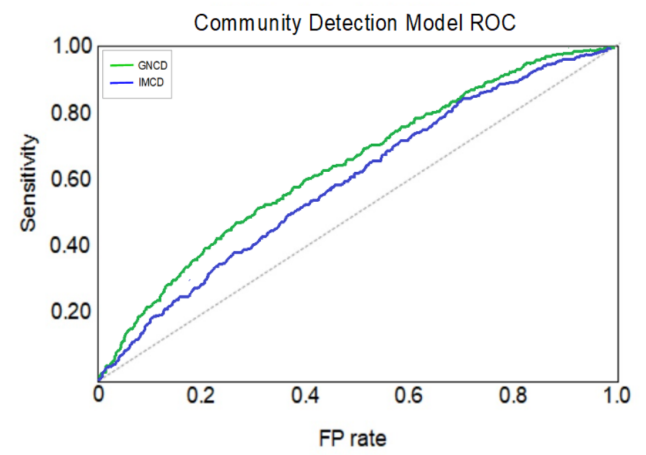

Figure 10: ROC Score

The number of correct and incorrect communities detected within the network were also measured using the confusion matrix rate, and a receiver operating characteristic (ROC) curve was plotted. The true positive (TP) rate, also known as sensitivity rate, is the proportion of the actual missing links that are predicted as such by the models. The FP rate (1-specificity) represents the proportion of the non-existing links that are identified as such by the models. However, the experimental results also seem to indicate that the Girvan-Newman Community Detection (GNCD) model has a higher level of accuracy than the Isomorphic Community Detection (IMCD) model, the GNCD takes a longer time to train with the same number of iterations. Furthermore the IMCD model was able to identify probable communities that were not detected by GNCD model.

\section{Discussion}

In this section we conducted an experiment on a wellstudied algorithm, the Girvan-Newman community detection model, for benchmarking purposes using the same dataset. We wanted to accomplish three goals, namely 1) compare the effectiveness of the proposed algorithm CCDAISM; 2) prove our hypothesis that it can detect community or communities that other algorithms miss and 3) open a new perspective by comparing the results of CCDA-ISM and traditional community detection methods, specifically the Girvan-Newman algorithm.

The Girvan-Newman community detection model is based on the notion of edge betweenness centrality that was investigated in the domain of SNA by Wasserman et al. [26]. The betweenness centrality value of an edge in a graph network is defined as the number of shortest paths between node-pairs traversing through that edge. Therefore, the betweenness of edges is a measurement of the flow of network activities that traverse along the edges of the graph. The fundamental notion of this model is that two tightly knit communities tend to be linked by edges where the network traffic traversing through should be relatively high [27].

The technique used in Girvan-Newman's algorithm involves the analysis and detection of community structure, depending on the repetitive elimination of edges, based on the highest number of the shortest paths that pass through them. By pruning or eliminating a certain edge, the mentioned network becomes clustered, divided, or segmented into smaller networks, i.e. communities or clusters of networks. The logic behind this algorithm was being able to find which edges in a network are anticipated most frequently between other pairs of nodes by discovering edges betweenness. The edges that have interconnections within the communities are expected to have high edge betweenness. The fundamental community structure of the network will be more fine-tuned once we eliminate edges with high edge betweenness.

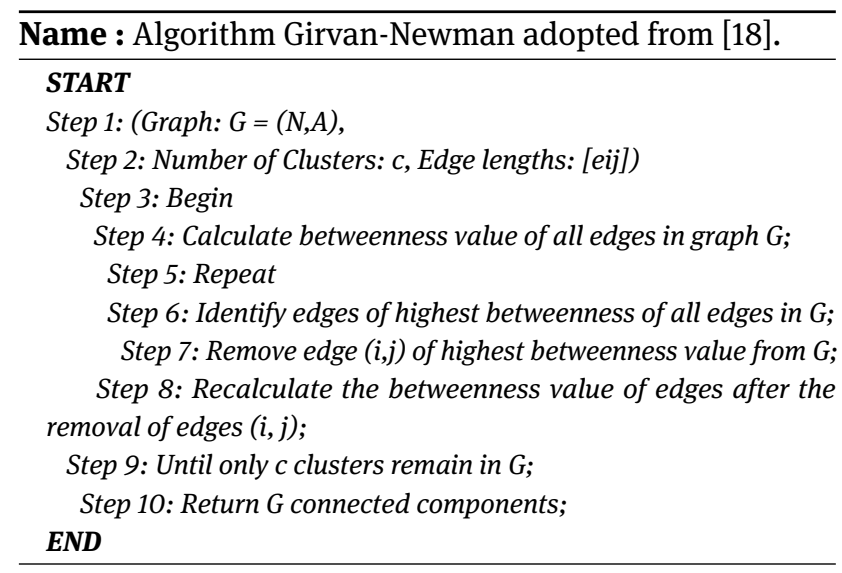

The edge betweenness $b(i, j)$ for edge e(i,j):

$$
B(i, j)=\sum_{i, k=s} \frac{\sigma i k e(i, j)}{\sigma i k}
$$

In Equation 1, * Where $\sigma i k$ is a total number of shortest paths from node $i$ to node $k$ and $\sigma i k e(i, j)$ is the number 
of those paths that pass through $e(i, j)$ and $s$ is the source node of path traversal.

Four (4) key communities` identified:

Table 3: The 4 key communities or cliques identified.

\begin{tabular}{ll}
\hline & Four key communities \\
\hline$\square \quad$ Grey \\
$\square \quad$ Red \\
$\square \quad$ Yellow \\
$\square \quad$ Green \\
$\square \quad$ Blue (Outliner Nodes) \\
\hline
\end{tabular}

Figure 11 represents the four key communities ${ }^{\star}$ or cliques identified. They are distinguished by the color of the node as represented in Table 3. Blue nodes are outliner nodes and not a community

\section{Isomorphic Subgraph Matching Results:}

It is found that the isomorphic subgraph matching model managed to identify a matching community 3 (nodes $3 \mathrm{~L}, 25 \mathrm{~S}, 27 \mathrm{M})$ in the community represented by red nodes.

Community 1 (nodes 32L,34L,40L) and 2 (nodes $14 \mathrm{~S}, 31 \mathrm{M}, 35 \mathrm{~L}$ ) detected via isomorphic subgraph only achieved partially with the communities detected based on the Girvan-Newman model as illustrated in the graph below:

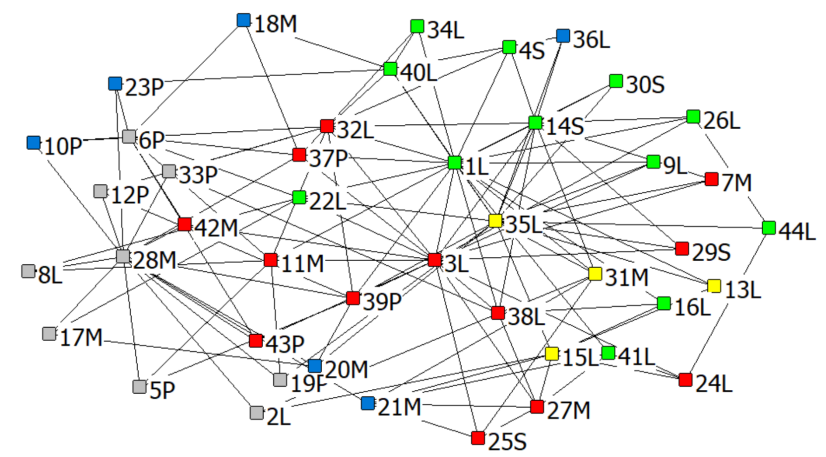

Figure 11: Four key communities* of the Cocaine Dealing Natarajan network as based on the Girvan-Newman community detection model, with a search depth of 20 .

Community 1 (Figure 6), Community 2 (Figure 7) and Community 3 (Figure 8) represent some of the isomorphic subgraph $\mathrm{P}$ that matches to base subgraph pattern $\mathrm{Q}$ (Figure 5). They were identified from our isomorphicbased community detection algorithm. (Figure 11) is the re- sult of the use of the Girvan-Newman algorithm, utilised for benchmarking purposes against the community detected using CCDA-ISM. As concluded in Table 2, CCDA-ISM can detect communities that overlap with the well-studied Girvan-Newman algorithm and can additionally detect isomorphic graphs of the community that may have gone undetected using traditional algorithms.

\section{Conclusion}

In this paper, we have put forward a community detection algorithm namely, CCDA-ISM and tested how effective it is in detecting communities using isomorphic subgraph matching. The CCDA-ISM algorithm can be very practical in real-life scenarios, as when a law enforcement teams engage in different ways of detecting a community. We also compared it to the well-studied algorithm GirvanNewman.

The results demonstrate that CCDA-ISM can detect communities which overlap with the well-studied GirvanNewman algorithm. This boosts confidence in the detection ability of CCDA-ISM algorithm, with the detected isomorphic graphs opening a new perspective in the domain of detecting community by incorporating subgraph isomorphic matching feature(s).

Future works that can be carried out following this research that is, Criminal network analysis (CNA) can include machine learning and deep reinforcement learning for detecting criminal communities [28] with more completeness, performance and accuracy. The criminal network prediction models commonly rely on Social Network Analysis (SNA) metrics. These models leverage on machine learning (ML) techniques to enhance the predictive accuracy of the models and processing speed [29], this can be a great scope to conduct research [30-34].

\section{References}

[1] Cai, Q., Ma, L., Gong, M., \& Tian, D., A survey on network community detection based on evolutionary computation. International Journal of Bio-Inspired Computation, Vol. 8(2), pp. 84-98, January, 2016.

[2] Lim, M., Abdullah, A., Jhanjhi, N., Khan, M. K., \& Supramaniam, M. (2019). Link Prediction in Time-Evolving Criminal Network With Deep Reinforcement Learning Technique. IEEE Access, 7, 184797-184807.

[3] Zhang, Chi, and Osmar R. Zaïane., Detecting local communities in networks with edge uncertainty, IEEE/ACM International Conference on Advances in Social Networks Analysis and Mining, 
Barcelona, Spain, 2018. pp. 9-16.

[4] Sangkaran, T., Abdullah, A., Jhanjhi, NZ., \& Supramaniam, M., Survey on isomorphic graph algorithms for graph analytics, International Journal of Computer Science and Network Security, Vol 19(1), pp. 85-92, January, 2019.

[5] Ullmann, J. R., An algorithm for subgraph isomorphism. Journal of the Association for Computer Machinery, Vol 23(1), pp.31-42, January, 1976.

[6] Cordella, L. P., Foggia, P., Sansone, C., \& Vento, M. Performance evaluation of the VF graph matching algorithm. IEEE Proceedings 10th International Conference on Image Analysis and Processing, Venice, Italy, pp. 1172-1177, September, 1999.

[7] Cordella, L. P., Foggia, P., Sansone, C., \& Vento, M.. A (sub) graph isomorphism algorithm for matching large graphs, IEEE Transactions on Pattern Analysis and Machine Intelligence, Vol 26(10), pp. 1367-1372, August 2004.

[8] Schmidt, D. C., \& Druffel, L. E., A fast backtracking algorithm to test directed graphs for isomorphism using distance matrices, Journal of the Association for Computer Machinery, Vol 23(3), pp. 433-445. July , 1976

[9] Corneil, D. G., \& Gotlieb, C. C., An efficient algorithm for graph isomorphism, Journal of the Association for Computer Machinery, Vol 17(1), pp.51-64, January, 1970

[10] Fu, L., \& Chandra, S., Optimized backtracking for subgraph isomorphism. International Journal of Database Management Systems, Vol 4(6), pp. 1. December, 2012.

[11] Javed, M. A., Younis, M. S., Latif, S., Qadir, J., \& Baig, A., Community detection in networks: A multidisciplinary review. Journal of Network and Computer Applications, Vol 108, pp. 87-111, April, 2018.

[12] Lim, M., Abdullah, A., \& Jhanjhi, N. Z. (2019). Performance optimization of criminal network hidden link prediction model with deep reinforcement learning. Journal of King Saud UniversityComputer and Information Sciences.

[13] M. Lim, A. Abdullah, NZ. Jhanjhi and M. K. Khan, " A Deep Reinforcement Learning Data Fusion model for Time-evolving Criminal Network Link Prediction," in IEEE Access. doi: 10.1109/ACCESS.2019.2958873, 2020.

[14] Li, J., Lear, M. J., Kawamoto, Y., Umemiya, S., Wong, A. R., Kwon, E., ... \& Hayashi, Y. Oxidative amidation of nitroalkanes with amine nucleophiles using molecular oxygen and iodine. Angewandte Chemie International Edition, Vol 54(44), pp. 1298612990, October, 2015.

[15] Newman, M. E., Community detection in networks: Modularity optimization and maximum likelihood are equivalent. arXiv preprint arXiv:1606.02319, June, 2016.

[16] Hu, R., Andreas, J., Rohrbach, M., Darrell, T., \& Saenko, K. Learning to reason: End-to-end module networks for visual question answering. IEEE International Conference on Computer Vision, Venice, Italy, 2017, pp. 804-813.

[17] Newman, M. E., \& Reinert, G., Estimating the number of communities in a network, Physical Review Letters, Vol. 117(7), pp. 078301, August, 2016.

[18] Krzakala, F., Moore, C., Mossel, E., Neeman, J., Sly, A., Zdeborová, L., \& Zhang, P., Spectral redemption in clustering sparse networks, Proceedings of the National Academy of Sciences, Vol.110(52), pp.20935-20940, December, 2013.
[19] Liu, C., \& Liu, Q., Community detection based on differential evolution using modularity density, Information 9, Vol (9), pp. 218, September, 2018.

[20] Magalingam, P., Davis, S., \& Rao, A., Using shortest path to discover criminal community, Digital Investigation, Vol. 15, pp. 1-17, December, 2015.

[21] Bahulkar, A., Szymanski, B. K., Baycik, N. O., \& Sharkey, T. C., Community detection with edge augmentation in criminal networks, IEEE/ACM International Conference on Advances in Social Networks Analysis and Mining, Barcelona, Spain,2018, pp. 11681175.

[22] Calderoni, F., \& Piccardi, C., Uncovering the structure of criminal organizations by community analysis: The infinito network, Tenth International Conference on Signal-Image Technology and Internet-Based Systems, Marrakech, Morocco, 2014, pp. 301-308

[23] Carletti, V., Foggia, P., Saggese, A., \& Vento, M., Challenging the time complexity of exact subgraph isomorphism for huge and dense graphs with VF3, IEEE Transactions on Pattern Analysis and Machine Intelligence, Vol 40(4), pp. 804-818, November, 2014.

[24] Omidvar, M. N., Li, X., Mei, Y., \& Yao, X. Cooperative co-evolution with differential grouping for large scale optimization. IEEE Transactions on Evolutionary Computation, Vol 18(3), pp. 378-393, September, 2013.

[25] Huang, Q., White, T., Jia, G., Musolesi, M., Turan, N., Tang, K., \& Yao, X., Community detection using cooperative co-evolutionary differential evolution, 2012 International Conference on Parallel Problem Solving from Nature, Berlin, Germany, pp. 235-244

[26] McKay, B. D., \& Piperno, A., Practical graph isomorphism, II. Journal of Symbolic Computation, Vol. 60, pp. 94-112, January, 2014.

[27] Dado, M., \& Bodemer, D. A review of methodological applications of social network analysis in computer-supported collaborative learning. Educational Research Review, Vol.22, pp. 159-180.

[28] Farine, Damien. The dynamics of transmission and the dynamics of networks. Journal of Animal Ecology 86, Vol.3, pp.415-418. May 2017

[29] Lim, M., Abdullah, A., Jhanjhi, NZ., \& Supramaniam, M., Hidden Link Prediction in Criminal Networks Using the Deep Reinforcement Learning Technique. Computers, Vol 8(1), pp 8, March, 2017

[30] Sangkaran, Theyvaa, Azween Abdullah, and N. Z. JhanJhi. "Criminal Network Community Detection Using Graphical Analytic Methods: A Survey." doi: 10.4108/eai.13-7-2018.162690

[31] Ozgul, F., Erdem, Z., Bowerman, C., \& Bondy, J. (2010, June). Combined detection model for criminal network detection. In Pacific-Asia Workshop on Intelligence and Security Informatics (pp. 1-14). Springer, Berlin, Heidelberg.

[32] Ozgul, F., Gok, M., Erdem, Z., \& Ozal, Y. (2012, June). Detecting criminal networks: SNA models are compared to proprietary models. In 2012 IEEE International Conference on Intelligence and Security Informatics (pp. 156-158). IEEE.

[33] Nowé, A., Vrancx, P., \& De Hauwere, Y. M. (2012). Game theory and multi-agent reinforcement learning. In Reinforcement Learning (pp. 441-470). Springer, Berlin, Heidelberg.

[34] Barbosa, S. E., \& Petty, M. D. (2014). Reinforcement learning in an environment synthetically augmented with digital pheromones. Advances in Artificial Intelligence, 2014. 